\title{
Spectra of helium clusters with up to six atoms using soft core potentials
}

\author{
M. Gattobigio, ${ }^{1}$ A. Kievsky, ${ }^{2}$ and M. Viviani ${ }^{2}$ \\ ${ }^{1}$ Université de Nice-Sophia Antipolis, \\ Institut Non-Linéaire de Nice, CNRS, \\ 1361 route des Lucioles, 06560 Valbonne, France \\ ${ }^{2}$ Istituto Nazionale di Fisica Nucleare, \\ Largo Pontecorvo 3, 56100 Pisa, Italy
}

\begin{abstract}
In this work we investigate small clusters of helium atoms using the hyperspherical harmonic basis. We consider systems with $A=2,3,4,5,6$ atoms with an inter-particle potential which does not present a strong repulsion at short distances. We use an attractive gaussian potential that reproduces the values of the dimer binding energy, the atom-atom scattering length, and the effective range obtained with one of the widely used He-He interactions, the LM2M2 potential. In systems with more than two atoms we consider a repulsive three-body force that, by construction, reproduces the trimer binding energy of the LM2M2 potential. With this model, consisting in the sum of a two- and three-body potential, we have calculated the spectrum of clusters formed by four, five and six helium atoms. We have found that these systems present two bound states, one deep and one shallow close to the threshold fixed by the energy of the $(A-1)$-atom system. Universal relations between the energies of the excited state of the $A$-atom system and the ground state energy of the $(A-1)$-atom system are extracted as well as the ratio between the ground state of the $A$-atom system and the ground state energy of the trimer.
\end{abstract}

PACS numbers: 31.15.xj, 31.15.xt, 36.90.+f, 34.10.+x 


\section{INTRODUCTION}

Systems composed by few helium atoms have been object of intense investigation from a theoretical and experimental point of view. The existence of the He-He molecule was experimentally established using diffraction experiments [1 14$]$. Its binding energy $E_{2 b}$ has been estimated to be around $1 \mathrm{mK}$ and its scattering length $a_{0}$ around 190 a.u. This makes the He-He molecule one of the biggest diatomic molecules. On the theoretical side, several He-He potentials have been proposed; in spite of different details and derivations, all of them share the common feature of a sharp repulsion below an inter-particle distance of approximately 5 a.u..

Another important characteristic of the He-He interactions is their effective range $r_{0} \approx$ 13 a.u.. Accordingly, the ratio $a_{0} / r_{0}$ is large enough $(>10)$ to place small helium clusters into the frame of Efimov physics [5, [6]. As shown by Efimov, when at least two of the two-body subsystems present an infinitely large scattering length (or zero binding energy) an infinite sequence of bound states (called Efimov states) appear in the three-body system; their binding energies scale in a geometrical way and they accumulate at zero energy. The scaling factor, $\mathrm{e}^{-2 \pi / s_{0}} \approx 1 / 515.03$, is universal and depends only on the ratio between particle masses (for three identical bosons $s_{0} \approx 1.00624$ ), not on the details of the two-body interaction (see Ref. [7] for a review). For a finite $a_{0} / r_{0}$ ratio, the number of the Efimov states has been estimated to be $N=\left(s_{0} / \pi\right) \ln \left|a_{0} / r_{0}\right|$ [6]; for example, the (bosonic) three ${ }^{4}$ He system presents an excited state just below the atom-dimer threshold that has been identified as an Efimov state.

Triggered by this interesting fact, several investigations of the helium trimer have been produced, establishing that its excited state is indeed an Efimov-like state (see for example Refs. [8 [10]). In addition, analysis of the atom-dimer collision in the ultracold regime have also been performed [11 13$]$.

One of the main difficulties in solving the quantum mechanical problem in the case of three helium atoms results to be the treatment of the strong repulsion at short distances of the He-He potential. Specific algorithms have been developed so far to solve this problem. The Faddeev equation has been opportunely modified [14]. Moreover, the hyperspheri-

cal adiabatic (HA) expansion has been extensively used in this case [13] (for a review see Ref. [15]). However, due to the difficulties in treating the strong repulsion, few calcula- 
tions exist for systems with more than three helium atoms. For example, in Ref. [16] the diffusion Monte Carlo method has been used to describe the ground state of He molecules up to 10 atoms, and in Ref. [17] a Monte Carlo technique has been used to construct the lowest adiabatic potential in systems with 3 and up to 10 helium atoms. On the other hand, description of few-atoms systems using soft-core potentials are currently operated (see for example Ref. [18]).

Therefore, the equivalence between hard- or soft-core-potential descriptions needs some clarification. In a recent work [19], an attractive He-He gaussian potential has been used to investigate the three ${ }^{4} \mathrm{He}$ system. In absence of direct experimental data, the two-body potential has been designed to reproduce the helium dimer binding energy $E_{2 b}$, the He-He scattering length $a_{0}$, and the effective range $r_{0}$ of the LM2M2 potential [20]. This gaussian potential can be considered as a regularized-two-body contact term in an Effective Field Theory (EFT) approximation of the physics driven by the LM2M2 potential [21]. It should be noticed that two potentials predicting similar values of $a_{0}$ and $r_{0}$ predict similar phase shifts in the low energy limit and, therefore, even if their shape is completely different, they describe in an equivalent way the physical processes in that limit [21]. The equivalence is lost as the energy is increased, when the details of the potential become more and more important.

Extending the study to the three-body system, differences between the attractive gaussian and the LM2M2 potentials are immediately observed. For example the trimer ground state energies differ by more than 15\% (see Table 【). A natural way to restore the equivalence between the two potentials is by the addition of a three-body soft-term force to the gaussian potential. On the other hand in an EFT treatment of the three-boson system with large scattering length, a three-body-contact term is required at leading order (LO). Its strength is usually determined by fixing a three-body observable as for example one of the trimer bound state energies. After this choice cut-off independent results are obtained [22]. Following this ideas, and based on Ref. [19], in the present work we have considered a gaussian-hypercentral three-body force with the strength fixed to reproduce the LM2M2 ground state binding energy of the three-atom system. The quality of this description has been studied for different ranges of the three-body force.

Using the two-atom and three-atom systems to fix the model interaction, we have analyzed heavier systems, up to $A=6$ atoms. The numerical calculations are performed by means of 
the hyperspherical harmonic ( $\mathrm{HH}$ ) expansion method with the technique developed recently by the authors in Ref. [23]. In this approach, the authors use the HH basis, without a previous symmetrization procedure, to describe bound states in systems up to six particles. The method is based in a particular representation of the Hamiltonian matrix, as a sum of products of sparse matrices, well suited for a numerical implementation. Converged results for different eigenvalues, with the corresponding eigenvectors belonging to different symmetries, have been obtained. As a novelty, in the present work we extend the formalism to treat a three-body force. Moreover, as we are dealing with atoms of ${ }^{4} \mathrm{He}$, only the the spectrum corresponding to totally symmetric eigenstates are of interest.

After fixing the strength of the three-body force to correctly describe the LM2M2 threebody ground state $E_{3 b}^{(0)}=126.4 \mathrm{mK}$, we have calculated the first three levels of the spectrum with total angular momentum $L=0$ of the $A=4,5,6$ systems. In the three cases we have found that the first two levels are bosonic bound states, one deep, $E_{A b}^{(0)}$, and one very shallow, $E_{A b}^{(1)}$, close to the threshold formed by the $A-1$ system plus one atom. The third state in all cases belongs to a mixed symmetry with an energy above the threshold and therefore not representing a bound state. The appearance of only two bound states in this systems is in agreement to previous calculations [17]. This fact has been observed in $A=4$ and interpreted as a consequence of the Efimov like spectrum of the $A=3$ system [24]. It should be noticed that, whereas converged results can be found in the literature for the ground state of the many atom systems, the energy of the excited states is much more difficult to calculate and only rough estimates are available.

To gain insight on the shallow state, we have varied the range of the three-body force (maintaining fixed the three-body ground state energy) and we have studied the effects of that variation in the $A=4,5,6$ spectrum. In the range considered, the variation produces small effects in the eigenvalues; however, it is crucial to determine if the shallow state is bound or not with respect to the $A-1$ threshold. Interestingly, we have observed that when the ranges of the two- and three-body forces have a ratio of about $\sqrt{2}$, the ratio between the shallow- and ground-state energy is $E_{A b}^{(1)} / E_{A-1 b}^{(0)} \approx 1.01-1.02$, in agreement with Refs. [25, 26]. This analysis confirms previous observations that each Efimov state in the $A=3$ system produces two bound states in the $A=4$ system. Furthermore, we have found $E_{4 b}^{(0)} / E_{3 b}^{(0)} \approx 4.5, E_{5 b}^{(0)} / E_{3 b}^{(0)} \approx 10.5$, and $E_{6 b}^{(0)} / E_{3 b}^{(0)} \approx 18.5$, which is in agreement with Refs. [26, 27]. 
The paper is organized as follows. In the next Section II we describe the two- and threebody forces we used in our calculations to reproduce LM2M2 data. In Section III the results for the bound states of the $A=3,4,5,6$ He clusters are collected whereas the conclusions are given in the last section. Some technical details of the method have been summarized in the Appendix.

\section{SOFT-CORE TWO- AND THREE-BODY HELIUM POTENTIAL}

As mentioned in the Introduction, the ${ }^{4} \mathrm{He}-{ }^{4} \mathrm{He}$ interaction presents a strong repulsion at short distances, below 5 a.u. This characteristic makes it difficult a detailed description of the system with more than four atoms. Accordingly, in the present work we have studied small clusters of helium interacting through a soft-core two- and three-body potentials which can be interpreted as regularized two- and three-body contact terms in a LO-EFT approximation of LM2M2.

Following Refs. [10, 19] we use the gaussian two-body potential

$$
V(r)=V_{0} \mathrm{e}^{-r^{2} / R^{2}}
$$

with $V_{0}=-1.227 \mathrm{~K}$ and $R=10.03$ a.u.. In the following we use $\hbar^{2} / m=43.281307$ (a.u.) $)^{2} \mathrm{~K}$. This parametrization of the two-body potential approximately reproduces the dimer binding energy $E_{2 b}$, the atom-atom scattering length $a_{0}$ and the effective range $r_{0}$ given by the LM2M2 potential. Specifically, the results for the gaussian potential are $E_{2 b}=-1.296 \mathrm{mK}$, $a_{0}=189.95$ a.u. and $r_{0}=13.85$ a.u., to be compared to the corresponding LM2M2 values $E_{2 b}=-1.302 \mathrm{mK}, a_{0}=189.05$ a.u. and $r_{0}=13.84$ a.u.. As shown in Ref. [19], the use of the gaussian potential in the three-atom system produces a ground state binding energy $E_{3 b}^{(0)}=150.4 \mathrm{mK}$, which is appreciable bigger than the LM2M2 helium trimer ground state binding energy of $126.4 \mathrm{mK}$. A smaller difference, though still appreciable, is observed in the first excited state (see Table I).

In order to have a closer description to the $A=3$ system obtained with the LM2M2 potential, we introduce the following three-body interaction

$$
W\left(\rho_{i j k}\right)=W_{0} \mathrm{e}^{-2 \rho_{i j k}^{2} / \rho_{0}^{2}},
$$

where $\rho_{i j k}^{2}=\frac{2}{3}\left(r_{i j}^{2}+r_{j k}^{2}+r_{k i}^{2}\right)$ is the three-body hyperradius in terms of the distances of the three interacting particles. Moreover, the strength $W_{0}$ is fixed to reproduce the LM2M2 
helium trimer binding energy of $126.4 \mathrm{mK}$. We have studied different cases by varying the range of the three-body force $\rho_{0}$ between 4 and 16 a.u.. Specific cases with the corresponding results in the $A=3$ system are shown in Table I. In the first two rows of the table we report the ground- and excited-binding energies of the trimer, both for LM2M2 potential and its gaussian representation. The excess of binding is evident for this last model. Successively, we report, for selected values of $W_{0}$ and $\rho_{0}$, the binding energies obtained summing to the gaussian potential the (repulsive) three-body force. By construction the ground state has been fixed to the LM2M2 value and, in addition, we can observe that the excited state $E_{3 b}^{(1)}$ is now closer to the corresponding LM2M2 result, showing an extremely small variation with $\rho_{0}$; the difference between the extremal values obtained for $\rho_{0}=4$ a.u. and $\rho_{0}=16$ a.u. is less than $1 \%$.

It should be noticed that the ranges $R$ of the two-body force and $\rho_{0}$ of the three-body force are somehow related. The gaussian two-body force can be thought as originating from a contact interaction regularized using a gaussian cutoff with $\Lambda=R^{-1}$ (see for example Ref. [7]). This means that configurations, in the $A=2$ system, in which two atoms have a relative momentum $k>\Lambda$ remain outside the present description or, in other words, details of the interaction for distances below $\sqrt{\left\langle r^{2}\right\rangle}=R / \sqrt{2}$ are not accessible. In the $A>2$ systems, three atoms interact through the three-body force when they happen to be inside a sphere of radius $\rho_{0} / \sqrt{2}$ at the same time. It is clear that, as no information is introduced in the two-body system for distances below $R / \sqrt{2}$, from the relation $\rho_{i j k}^{2}=\frac{2}{3}\left(r_{i j}^{2}+r_{j k}^{2}+r_{k i}^{2}\right)$ and putting each distance at the value $R / \sqrt{2}$, we obtain for the three-body range $\rho^{2}=R^{2}$. Since $R$ has been fixed in order to describe two-body quantities, in the description of systems with $A>3$ atoms we consider different values of $\rho_{0}$ with particular attention at the region $\rho_{0} \approx \sqrt{2} R$.

The calculations for the $A \geq 3$ systems, up to six atoms, are performed using the unsymmetrized HH basis. The method has been recently used to describe up to six nucleons interacting through a central potential [23]. A brief description of the method is given in the Appendix. The novelty presented here regards the implementation of the three-body force. Using a particular rotation of the $\mathrm{HH}$ basis it is possible to construct the potential energy as a product of sparse matrices. The Hamiltonian matrix is obtained using the following 
orthonormal basis

$$
\langle\rho \Omega \mid m[K]\rangle=\left(\beta^{(\alpha+1) / 2} \sqrt{\frac{m !}{(\alpha+m) !}} L_{m}^{(\alpha)}(\beta \rho) \mathrm{e}^{-\beta \rho / 2}\right) \mathcal{Y}_{[K]}^{L M}\left(\Omega_{N}\right),
$$

where $L_{m}^{(\alpha)}(\beta \rho)$ is a Laguerre polynomial with $\alpha=3 N-1$ and $\beta$ a variational non-linear parameter. The matrix elements of the Hamiltonian are obtained after integrations in the $\rho, \Omega$ spaces. They depend on the indices $m, m^{\prime}$ and $[K],\left[K^{\prime}\right]$ as follows

$$
\begin{array}{r}
\left\langle m^{\prime}\left[K^{\prime}\right]|H| m[K]\right\rangle=-\frac{\hbar^{2} \beta^{2}}{m}\left(T_{m^{\prime} m}^{(1)}-K(K+3 N-2) T_{m^{\prime} m}^{(2)}\right) \delta_{\left[K^{\prime}\right][K]} \\
+\sum_{i<j}\left[\sum_{\left[K^{\prime \prime}\right]\left[K^{\prime \prime \prime}\right]} \mathcal{B}_{[K]\left[K^{\prime \prime}\right]}^{i j, L M} \mathcal{B}_{\left[K^{\prime \prime \prime}\right]\left[K^{\prime}\right]}^{i j, L M} V_{\left[K^{\prime \prime}\right]\left[K^{\prime \prime \prime}\right]}^{\left.m, m^{\prime}\right]}\right]+\sum_{i<j<k}\left[\sum_{\left[K^{\prime \prime}\right]\left[K^{\prime \prime \prime}\right]} \mathcal{D}_{[K]\left[K^{\prime \prime}\right]}^{i j k, L M} \mathcal{D}_{\left[K^{\prime \prime \prime}\right]\left[K^{\prime}\right]}^{i j j, L M} W_{\left[K^{\prime \prime}\right]\left[K^{\prime \prime \prime}\right]}^{m, m^{\prime}}\right] .
\end{array}
$$

The matrices $T^{(1)}$ and $T^{(2)}$ have an analytical form and are given in Ref. [28]. The matrix elements $V_{[K]\left[K^{\prime}\right]}^{m, m^{\prime}}$ and $W_{[K]\left[K^{\prime}\right]}^{m, m^{\prime}}$ are obtained after integrating the matrices $V_{12}(\rho)$ and $W(\rho)$ in $\rho$-space (we will call the corresponding matrices $V_{12}$ and $W$ ). Introducing the diagonal matrix $D$ such that $\left\langle\left[K^{\prime}\right]|D|[K]\right\rangle=\delta_{[K],\left[K^{\prime}\right]} K(K+3 N-2)$, and the identity matrix $I$ in $K$-space, we can rewrite the Hamiltonian schematically as

$$
H=-\frac{\hbar^{2} \beta^{2}}{m}\left({ }^{(1)} T \otimes I+{ }^{(2)} T \otimes D\right)+\sum_{i<j}\left[\mathcal{B}_{i j}^{L M}\right]^{t} V_{12} \mathcal{B}_{i j}^{L M},+\sum_{i<j<k}\left[\mathcal{D}_{i j k}^{L M}\right]^{t} W \mathcal{D}_{i j k}^{L M},
$$

in which the tensor product character of the kinetic energy is explicitly given. A method to diagonalize a matrix of this form is given in Ref. [23].

\section{RESULTS FOR $A=4,5,6$ HE CLUSTERS}

In this sections we present results for small clusters, up to $A=6$, formed by atoms of ${ }^{4}$ He. Despite the differences observed at the level of the three-body system between the gaussian two-body force and the LM2M2 potential, it is of interest the computation of the spectrum produced by the gaussian two-body force only for the $A=4,5,6$ systems.

In Table [1] we show the $L=0$ ground state $E_{A b}^{(0)}$ and the first two excited states $E_{A b}^{(1)}$ and $E_{A b}^{(2)}$ for increasing values of the grand-angular momentum $K$ using the unsymmetrized HH basis. The calculations have been performed up to $K=40$ in $A=4, K=24$ in $A=5$ and $K=22$ in $A=6$. It is a property of the $\mathrm{HH}$ basis that when all states having a fixed value of $K$ are included in the expansion of the wave function, the symmetry of 
the eigenvectors reflects the symmetries present in the Hamiltonian. Since the Hamiltonian is symmetric under exchange of the particles, the obtained eigenvectors have well defined particle permutation symmetry. In the present case the ground state $E_{A b}^{(0)}$ and first excited state $E_{A b}^{(1)}$ of the Hamiltonian matrix for $A=4,5,6$ are symmetric states and belong to the irreducible representation $[\boldsymbol{\lambda}]$ with $\boldsymbol{\lambda}=\boldsymbol{A}$. In all cases the second excited state $E_{A b}^{(2)}$ has mixed symmetry and belongs to the irreducible representation $[\boldsymbol{\lambda} 1]$ with $\boldsymbol{\lambda}=\boldsymbol{A}-\mathbf{1}$. In Table I we also observe that the ground state binding energy, $E_{A b}^{(0)}$, has a very fast convergence in terms of $K$ and can be determined with five digits; this value fixes the threshold of the continuum spectrum in the $A+1$ system. True bound states in the $A=4$ systems are those having a binding energy bigger than the trimer binding energy of $150.4 \mathrm{mK}$ and, looking at the table, bound states in the $A=5,6$ systems appears below the threshold of $751.38 \mathrm{mK}$ and $1945.2 \mathrm{mK}$ respectively. Since in all cases the second excited state $E_{A b}^{(2)}$ results to be above the threshold, only two bosonic states are bound in the $A=4,5,6$ systems, one deep and one shallow close to the $A-1$ threshold. The next bosonic state appears above $E_{A b}^{(2)}$ and, therefore, it is not bound. This result confirms previous analysis in the four body sector that the lower Efimov state in the $A=3$ system produces two bound states, one deep and one shallow. Here, we have extended this observation up to the $A=6$ system. The convergence of the $E_{A b}^{(1)}$ is much slower than for the ground state, however with the extended based used it has been determined with an accuracy well below $1 \%$.

For this atom-atom potential the ratio $r_{0} / a \approx 1 / 14$ and therefore we are not too far from the unitary limit, and we can make predictions for the universal ratios $E_{A b}^{(1)} / E_{(A-1) b}^{(0)}$ and $E_{A b}^{(0)} / E_{3 b}^{(0)}$. From the table we can observe that $E_{4 b}^{(1)} / E_{3 b}^{(0)}=1.085, E_{5 b}^{(1)} / E_{4 b}^{(0)}=1.10$ and $E_{6 b}^{(1)} / E_{5 b}^{(0)}=1.13$. These results are not so close to the universal ratio of around 1.01 indicating that effective range corrections are important. For the ratios with respect to the trimer ground state we have, $E_{4 b}^{(0)} / E_{3 b}^{(0)}=5.01, E_{5 b}^{(0)} / E_{3 b}^{(0)}=12.97$ and $E_{6 b}^{(0)} / E_{3 b}^{(0)}=25.4$. As we will see, these ratios are substantially modified when a three-body force is included.

Now, we consider the model with both two- and three-body interaction. The pattern of convergence for the ground and excited states of the $A=4,5,6$ helium systems, using the gaussian two-body potential plus the repulsive three-body potential with $\rho_{0}=14$ a.u., is given in Table III. The maximum grand angular momentum considered is $K=40$ for $A=4$, $K=24$ for $A=5$ and $K=22$ for $A=6$. As in the case in which only the two-body force has been considered, in all of the three cases only two bound states appears, one deep and 
one shallow very close to the $A-1$ threshold. The ground state presents a fast convergence with $K$ and the accuracy can be estimate below $0.1 \mathrm{mK}$. The convergence for the excited state is slower and, for the values of $K$ considered, its accuracy is given at the level of $3 \mathrm{mK}$. However, from the results it is well established that, with the value of $\rho_{0}$ considered, the excited state, $E_{A b}^{(1)}$, is bound with respect to the $A-1$ threshold. In fact, for $A=4$ the $3+1$ threshold appears at $126.4 \mathrm{mK}$ and the upper bound estimate for this state is 129 $\mathrm{mK}$. Its ratio $E_{4 b}^{(1)} / E_{3 b}^{(0)}$ is 1.020 . For $A=5$ the $4+1$ threshold appears at $568.8 \mathrm{mK}$ and the upper bound estimate for the excited state is $575 \mathrm{mK}$. Its ratio $E_{5 b}^{(1)} / E_{4 b}^{(0)}$ is 1.011. For $A=6$ the $5+1$ threshold appears at $1326.6 \mathrm{mK}$ and the upper bound estimate for the excited state is $1350 \mathrm{mK}$. Its ratio $E_{6 b}^{(1)} / E_{5 b}^{(0)}$ is 1.018 . The ratio between the trimer ground state and the ground states of the $A=4,5,6$ systems are $E_{4 b}^{(0)} / E_{3 b}^{(0)}=4.5, E_{5 b}^{(0)} / E_{3 b}^{(0)}=10.5$ and $E_{5 b}^{(0)} / E_{3 b}^{(0)}=18.5$, respectively. These ratios are in good agreement with those given in Refs. [25 27], and represent a substantial improvement with respect to the case in which the three-body force is not included. At the ratio $r_{0} / a$ under consideration the use of the two-body soft-core potential alone reduces the Efimov character of ground and first-exited states, which is recovered by including the three-body force. It is interesting to compare the results obtained using the soft-core representation of the LM2M2 potential with the results of Refs. [16, 17] (quoted in Table III) obtained using the original LM2M2 interaction. For the ground state the agreement is around $2 \%$ for $A=4,5$ and around $1 \%$ for $A=6$. The agreement is worst for the excited state, however the results from Ref. [17] are obtained using approximate solutions of the adiabatic hyperspherical equations.

The overall agreement for $A=4,5,6$ between LM2M2 and soft potential gives a further indication that at the LO in an EFT approach to the Efimov physics there is no need of a four-body force; this is only a side observation which is not at all conclusive for the lack of systematic study as a function of the cut-off.

Moreover, in the four panels of Fig. 2 we analyze modifications to the spectrum of the systems we have considered when different values of $W_{0}$ and $\rho_{0}$ are used. The results for $A=3$ can be extracted from Table III; the $A=3$ ground state is stable by construction, and small variations are observed for $E_{3 b}^{(1)}$. As shown in Fig. $2 \mathrm{a}, E_{3 b}^{(1)}$ is always below the $2+1$ threshold. For $A=4$, see Fig. 2 b, the excited state $E_{4 b}^{(1)}$ is above the $3+1$ threshold, and therefore not bounded for values of $\rho_{0}<7$ a.u. For $A=5$, Fig. 2r, and $A=6$, Fig. 2d, the corresponding excited states are above the $4+1$ and $5+1$ thresholds for for values of 
$\rho_{0}<12$ a.u., and $\rho_{0}<10$ a.u., respectively. For $A=5,6$ the results for the bound state present a bump with the smaller binding energy around $\rho_{0}=10$ a.u.. To sum up, the most sensitive property of the spectrum as a function of $\rho_{0}$ is, for $A=4,5,6$, if the $E_{A b}^{(1)}$ is above or below threshold. As previously discussed, a reasonable choice is $\rho_{0}=14$ a.u., and around this value all the excited states are bound.

Finally, in Table IV the results for the universal ratios are shown in for values of $\rho_{0}=$ $12,14,16$ a.u.; we observe that small variation of $\rho_{0}$ do not drastically change these values. It should be noticed that in the present analysis the unitary limit is not completely reached since the ratio between the two-body effective range and scattering length is $r_{0} / a \approx 1 / 14$. An analysis of the universal ratios as a function of $a$ is in progress.

\section{CONCLUSIONS}

In this work we have attached two different problems. From one side, we have studied the possibility of calculating bound and excited states in a bosonic (atomic) system up to $A=6$ using the unsymmetrized HH expansion and considering soft two- and three-body forces. On the other hand, the model has been constructed to approximate the description of small helium clusters taking as a reference the results of the LM2M2 potential. These two problems are related since the LM2M2 presents a strong repulsion at short distances. Therefore, the possibility of using a soft-core representation of the original potential has been analyzed in detail. In Ref. [19] bound states and low energy scattering states of the trimer have been analyzed using the soft-core representation of the LM2M2. The results obtained in that work were encouraging in the sense that they were found to be in close agreement to those obtained using the original potential.

Here, we have extended the analysis to bigger systems. Therefore, the description of such systems with sufficient accuracy is of the main importance. To this end, we have used a method recently developed in Ref. [23] in which the HH basis is used without symmetrization of the basis states. The basis is complete and, when all basis elements are included up to a certain maximum value of the grand angular momentum $K$, the eigenvectors reflect the symmetries present in the Hamiltonian. In the particular case here considered, the eigenvectors have well defined symmetry under particle permutation and they can be organized as belonging to the different irreducible representations of the group of permutations 
of $A$ objects, $S_{A}$. This simple fact has allowed to make an important statement regarding the number of bosonic bound states present in the systems under study. After the direct diagonalization of the $A$-body system we have analyzed the first three states for increasing values of $K$. We have considered very extended basis, up to $K=40$ for the $A=4$ system and $K=24(K=22)$ for the $A=5(A=6)$ system. This allowed to obtain converged results for the first eigenvalues of the spectrum. The first two were symmetric states having eigenvalues with energy below the continuum threshold (fixed by the lowest bound state in the $A-1$ system) and therefore they represent true bound states. The third state was found to belong to a mixed symmetry and results to be above the threshold. This was the case for all the systems considered $(A=4,5,6)$ and it means that the next bosonic state has an energy above the mixed state and therefore it is not bound. Therefore we have unambiguously determined that these systems present only two bound states.

The two bosonic bound states has been studied for different values of the three-body potential rage $\rho_{0}$. This analysis is given in Fig. 2 where the position of the excited state moves from unbound to bound as $\rho_{0}$ increases. The particular case $\rho_{0} \approx \sqrt{2} R$ is explicitly given in Table $\amalg$ showing that in fact the excited state is slightly bound. Moreover, since the He-He potential predicts a large two-body scattering length, we have studied the universal ratios $E_{A b}^{(0)} / E_{3 b}^{(0)}$ and $E_{A b}^{(1)} / E_{(A-1) b}^{(0)}$. These ratios have been studied in detail in the $A=4$ case (see Refs. [24 26]). Estimates have also been obtained for bigger systems [27]. Our calculations, obtained for one particular value of the ratio $r_{0} / a$, are in agreement with those references on the universal character of these states in $A>3$ systems. An analysis of the universal ratios as $a \rightarrow \infty$ is at present under way.

Finally, we would like to discuss the quality of the description using the two- and threebody soft-core-potential model. We observe a substantial good agreement, at the level of $2 \%$ or better, for the ground states of the $A$-atom systems in comparison to the results of the LM2M2 potential given by Lewerenz (Ref. [16]). The excited states have been calculated in Ref. [17] though using a reduced Hilbert space. Comparing to those results we observe an agreement around 5\%. From this analysis we can conclude that a four-body force will have effects beyond this level of accuracy. A deeper analysis in this subject is in progress. 


\section{APPENDIX}

Following Refs. [23, 28], we present a brief overview of the properties of the HH basis and its implementation without generating basis elements with well defined permutational symmetry. This approach allows to avoid the complications of symmetry-adapted-basis construction, and to easily treat permutational-symmetry-breaking terms [23, 29]

We start with the following definition of the Jacobi coordinates for an equal mass $A$ body system with Cartesian coordinates $\mathbf{r}_{1} \ldots \mathbf{r}_{A}$

$$
\mathbf{x}_{N-j+1}=\sqrt{\frac{2 j}{j+1}}\left(\mathbf{r}_{j+1}-\mathbf{X}_{j}\right), \quad j=1, \ldots, N .
$$

For a given set of Jacobi coordinates $\mathbf{x}_{1}, \ldots, \mathbf{x}_{N}$, we can introduce the hyperradius $\rho$

$$
\rho=\left(\sum_{i=1}^{N} x_{i}^{2}\right)^{1 / 2}=\left(2 \sum_{i=1}^{A}\left(\mathbf{r}_{i}-\mathbf{X}\right)^{2}\right)^{1 / 2}=\left(\frac{2}{A} \sum_{j>i}^{A}\left(\mathbf{r}_{j}-\mathbf{r}_{i}\right)^{2}\right)^{1 / 2}
$$

and the hyperangular coordinates $\Omega_{N}$

$$
\Omega_{N}=\left(\hat{\boldsymbol{x}}_{1}, \ldots, \hat{\boldsymbol{x}}_{N}, \phi_{2}, \ldots, \phi_{N}\right)
$$

with the hyperangles $\phi_{i}$ defined via

$$
\begin{aligned}
x_{N}= & \rho \cos \phi_{N} \\
x_{N-1} & =\rho \sin \phi_{N} \cos \phi_{N-1} \\
& \vdots \\
x_{i}= & \rho \sin \phi_{N} \cdots \sin \phi_{i+1} \cos \phi_{i} \\
& \vdots \\
x_{2}= & \rho \sin \phi_{N} \cdots \sin \phi_{3} \cos \phi_{2} \\
x_{1}= & \rho \sin \phi_{N} \cdots \sin \phi_{3} \sin \phi_{2} .
\end{aligned}
$$

The explicit expression for the $\mathrm{HH}$ functions, having well defined values of $L M$, is

$\mathcal{Y}_{[K]}^{L M}\left(\Omega_{N}\right)=\left[\prod_{j=2}^{N} \mathcal{P}_{K_{j}}^{\alpha_{l_{j}}, \alpha_{K_{j-1}}}\left(\phi_{j}\right)\right]\left[\left.\left.Y_{l_{1}}\left(\hat{\boldsymbol{x}}_{1}\right) \otimes Y_{l_{2}}\left(\hat{\boldsymbol{x}}_{2}\right)\right|_{L_{2}} \ldots \otimes Y_{l_{N-1}}\left(\hat{\boldsymbol{x}}_{N-1}\right)\right|_{L_{N-1}} \otimes Y_{l_{N}}\left(\hat{\boldsymbol{x}}_{N}\right)\right]_{L M}$,

with the indicated coupling scheme. The hyperspherical polynomial is

$$
\mathcal{P}_{K_{j}}^{\alpha_{l_{j}}, \alpha_{K_{j-1}}}\left(\phi_{j}\right)=\mathcal{N}_{n_{j}}^{\alpha_{l_{j}}, \alpha_{K_{j}}}\left(\cos \phi_{j}\right)^{l_{j}}\left(\sin \phi_{j}\right)^{K_{j-1}} P_{n_{j}}^{\alpha_{K_{j-1}}, \alpha_{l_{j}}}\left(\cos 2 \phi_{j}\right) .
$$


The set of quantum numbers $[K]$ includes the $n_{2} \ldots n_{N}$ indices of the Jacobi polynomials, the $l_{1} \ldots l_{N}$ angular momenta of the particles and the intermediate couplings $L_{2} \ldots L_{N-1}$. The $K_{j}$ quantum numbers are defined as

$$
K_{j}=\sum_{i=1}^{j}\left(l_{i}+2 n_{i}\right), \quad n_{1}=0, \quad K \equiv K_{N},
$$

$K \equiv K_{N}$ is known as the grand angular momentum, and $\mathcal{N}_{n}^{\alpha \beta}$ is a normalization factor. For the definition of the $\alpha_{a}$, where $a$ can be either an angular momentum $l_{j}$ or a quantum number $K_{j}$, one needs to introduce the hyperspherical-binary-tree structure [30]. For example the tree of Fig. 1 corresponds to the choice of hyperangles given by Eq. (9), in which the coefficients specializes to $\alpha_{K_{j}}=K_{j}+3 j / 2-1$ and $\alpha_{l_{j}}=l_{j}+1 / 2$.

Hyperspherical functions constructed using different hyperspherical-coordinate definitions can be related using the $\mathcal{T}$-coefficients [31, 32]. Schematically, these coefficients relate the following tree structures
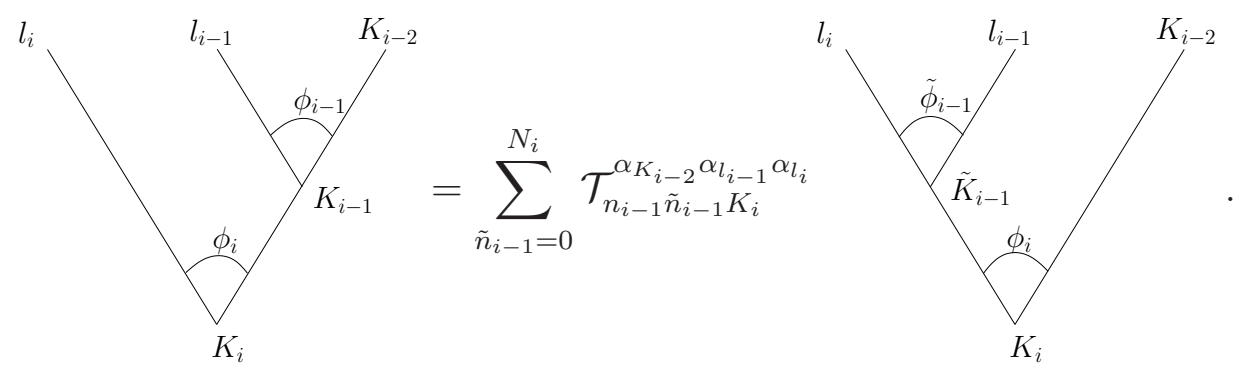

Here $K_{i}=K_{i-1}+l_{i}+2 n_{i}=\tilde{K}_{i-1}+l_{i}+2 \tilde{n}_{i}$ The explicit definition of the coefficients is given in Ref. [23]. Let us call $\mathcal{Y}_{[K]}^{L M}\left(\Omega_{N}^{i}\right)$ the HH basis element constructed in terms of a set of Jacobi coordinates in which the $i$-th and $i+1$-th Jacobi vectors results from the transposition between particles $j, j+1$

$$
\begin{aligned}
\mathbf{x}_{i}^{\prime} & =-\frac{1}{j} \mathbf{x}_{i}+\frac{\sqrt{(j+1)^{2}-2(j+1)}}{j} \mathbf{x}_{i+1} \\
\mathbf{x}_{i+1}^{\prime} & =\frac{\sqrt{(j+1)^{2}-2(j+1)}}{j} \mathbf{x}_{i}+\frac{1}{j} \mathbf{x}_{i+1},
\end{aligned}
$$

with all the other vectors equal to the original ones (transposed basis). The coefficients

$$
\mathcal{A}_{[K]\left[K^{\prime}\right]}^{i, L M}=\int d \Omega_{N}\left[\mathcal{Y}_{[K]}^{L M}\left(\Omega_{N}\right)\right]^{*} \mathcal{Y}_{\left[K^{\prime}\right]}^{L M}\left(\Omega_{N}^{i}\right)
$$

are the matrix elements of a matrix $\mathcal{A}_{i}^{L M}$ that allows to express the transposed HH basis elements in terms of the reference basis. The coefficients $\mathcal{A}_{[K]\left[K^{\prime}\right]}^{i, L M}$ form a very sparse matrix 
and they can be calculated analytically using the $\mathcal{T}$ - coupling coefficients and the RaynalRevai matrix elements [23] . A generic rotation between the reference $\mathrm{HH}$ basis and a basis in which the last Jacobi vector is defined as $\mathbf{x}_{N}^{\prime}=\mathbf{r}_{j}-\mathbf{r}_{i}$ can be constructed as successive products of the $\mathcal{A}_{[K]\left[K^{\prime}\right]}^{k, L M}$ coefficients. Defining $\mathcal{Y}_{[K]}^{L M}\left(\Omega_{N}^{i j}\right)$ the HH basis element constructed in terms of a set of Jacobi coordinates in which the $N$-th Jacobi vector is defined $\mathbf{x}_{N}^{\prime}=\mathbf{r}_{j}-\mathbf{r}_{i}$, this coefficient can be given in the following form

$$
\mathcal{B}_{[K]\left[K^{\prime}\right]}^{i j, L M}=\int d \Omega\left[\mathcal{Y}_{[K]}^{L M}\left(\Omega_{N}\right)\right]^{*} \mathcal{Y}_{[K]}^{L M}\left(\Omega_{N}^{i j}\right)=\left[\mathcal{A}_{i_{1}}^{L M} \cdots \mathcal{A}_{i_{n}}^{L M}\right]_{[K]\left[K^{\prime}\right]}
$$

The particular values of the indices $i_{1}, \ldots, i_{n}$, labelling the matrices $\mathcal{A}_{i_{1}}^{L M}, \ldots, \mathcal{A}_{i_{n}}^{L M}$, depend on the pair $(i, j)$. The matrix

$$
\mathcal{B}_{i j}^{L M}=\mathcal{A}_{i_{1}}^{L M} \cdots \mathcal{A}_{i_{n}}^{L M}
$$

is written as a product of the sparse matrices $\mathcal{A}_{i}^{L M}$ 's.

We consider now the potential energy of an $A$-body system constructed in terms of twobody interactions

$$
V=\sum_{i<j} V(i, j)
$$

In terms of the $\mathrm{HH}$ basis it results

$$
\sum_{i j} V_{i j}(\rho)=\sum_{i j}\left[\mathcal{B}_{i j}^{L M}\right]^{t} V_{12}(\rho) \mathcal{B}_{i j}^{L M}
$$

where the matrix elements of the matrix $V_{12}(\rho)$ are defined as

$$
\begin{aligned}
& V_{[K]\left[K^{\prime}\right]}^{(1,2)}(\rho)=\left\langle\mathcal{Y}_{[K]}^{L M}\left(\Omega_{N}\right)|V(1,2)| \mathcal{Y}_{\left[K^{\prime}\right]}^{L M}\left(\Omega_{N}\right)\right\rangle= \\
& \delta_{l_{1}, l_{1}^{\prime}} \cdots \delta_{l_{N}, l_{N}^{\prime}} \delta_{L_{2}, L_{2}^{\prime}} \cdots \delta_{L_{N}, L_{N}^{\prime}} \delta_{K_{2}, K_{2}^{\prime}} \cdots \delta_{K_{N}, K_{N}^{\prime}} \\
& \times \int d \phi_{N}\left(\cos \phi_{N} \sin \phi_{N}\right)^{2} \mathcal{P}_{K_{N}}^{\alpha_{l_{N}}, \alpha_{K_{N-1}}}\left(\phi_{N}\right) V\left(\rho \cos \phi_{N}\right) \mathcal{P}_{K_{N}^{\prime}}^{\alpha_{l_{N}}, \alpha_{K_{N-1}}}\left(\phi_{N}\right)
\end{aligned}
$$

Each term of the sum in Eq.(19) results in a product of sparse matrices, a property which allows an efficient implementation of matrix-vector product. This procedure can be easily extended to spin-dependent potentials [33].

We now consider a three body force depending on the hyperradius $\rho_{i j k}$ of a triplet of particles $\mathbf{r}_{i}, \mathbf{r}_{j}, \mathbf{r}_{k}$,

$$
V^{(3)}=\sum_{i<j<k} W\left(\rho_{i j k}\right)
$$


The term in which $i, j, k \equiv 1,2,3$ verifies $\rho_{123}^{2}=x_{N}^{2}+x_{N-1}^{2}$. It can be calculated on a hyperspherical-basis set relative to an non-standard hyperspherical tree with the branches attached to leaves $x_{N}$ and $x_{N-1}$ going to the same node. The transition between the two hyperspherical sets is given by the $\mathcal{T}$-coefficients

$$
\mathcal{Y}_{[K]}^{L M}\left(\Omega_{N}\right)=\sum_{\tilde{n}_{N-1}} \mathcal{T}_{n_{N-1} \tilde{n}_{N-1} K}^{\alpha_{K_{N-2}} \alpha_{l_{N-1}} \alpha_{l_{N}}} \mathcal{Y}_{[\tilde{K}]}^{L M}\left(\tilde{\Omega}_{N}\right)
$$

where all the variable with the tilde refer to the non-standard tree. With this choice we simply have

$$
\rho_{123}=\rho \cos \phi_{N}
$$

and the fixed-rho matrix elements reads

$$
\begin{aligned}
& \left\langle\mathcal{Y}_{\left[\tilde{K}^{\prime}\right]}^{L M}\left(\tilde{\Omega}_{N}\right)|W(\rho)| \mathcal{Y}_{[\tilde{K}]}^{L M}\left(\tilde{\Omega}_{N}\right)\right\rangle=\delta_{l_{1}^{\prime}, l_{1}} \cdots \delta_{l_{N}^{\prime}, l_{N}} \delta_{L_{2}^{\prime}, L_{2}} \cdots \delta_{L^{\prime}, L} \delta_{M^{\prime}, M} \delta_{\tilde{K}_{2}^{\prime}, \tilde{K}_{2}} \cdots \delta_{\tilde{K}_{N-1}^{\prime}, \tilde{K}_{N-1}} \\
& \int\left(\cos \phi_{N}\right)^{4}\left(\sin \phi_{N}\right)^{3 N-8} d \phi_{N} \mathcal{P}_{K^{\prime}}^{\alpha_{\tilde{K}_{N-1}}, \alpha_{K_{N-2}}}\left(\phi_{N}\right) \mathcal{P}_{K}^{\alpha_{\tilde{K}_{N-1}}, \alpha_{K_{N-2}}}\left(\phi_{N}\right) W\left(\rho \cos \phi_{N}\right) .
\end{aligned}
$$

The three-body force matrix $W(\rho)$ is extremely sparse, and it is diagonal on all quantum numbers but the grand-angular momentum. Finally the matrix $W(\rho)$ in the standard basis is obtained by means of the $\mathcal{T}$-coefficients

$$
\left\langle\mathcal{Y}_{\left[K^{\prime}\right]}^{L M}\left(\Omega_{N}\right)|W(\rho)| \mathcal{Y}_{[K]}^{L M}\left(\Omega_{N}\right)\right\rangle=\sum_{\tilde{n}_{N-1}} \mathcal{T}_{n_{N-1}^{\prime} \tilde{n}_{N-1} K^{\prime}}^{\alpha_{K_{N-2}} \alpha_{l_{N-1}} \alpha_{l_{N}}} \mathcal{T}_{n_{N-1} \tilde{n}_{N-1} K}^{\alpha_{K_{N-2}} \alpha_{l_{N-1}} \alpha_{l_{N}}}\left\langle\mathcal{Y}_{\left[\tilde{K}^{\prime}\right]}^{L M}\left(\tilde{\Omega}_{N}\right)\left|W\left(\rho_{123}\right)\right| \mathcal{Y}_{[\tilde{K}]}^{L M}\left(\tilde{\Omega}_{N}\right)\right\rangle
$$

In order to calculate the other terms of the three-body force, we use the matrices $\mathcal{A}_{p}^{L M}$, defined in Eq. (15), that transpose particles; with a suitable product of these sparse matrices

$$
\mathcal{D}_{i j k}^{L M}=\mathcal{A}_{p_{1}}^{L M} \cdots \mathcal{A}_{p_{m}}^{L M}
$$

we can permute the particles in such a way that $\mathbf{x}_{N}=\mathbf{r}_{i}-\mathbf{r}_{j}$, and $\mathbf{x}_{N-1}=2 / \sqrt{3}\left(\mathbf{r}_{k}-\left(\mathbf{r}_{i}+\right.\right.$ $\left.\mathbf{r}_{j}\right) / 2$ ), and $\rho_{i j k}^{2}=x_{N-1}^{2}+x_{N}^{2}$, and the total three-body force reads

$$
V^{(3)}=\sum_{i<j<k}\left[\mathcal{D}_{i j k}^{L M}\right]^{t} W(\rho) \mathcal{D}_{i j k}^{L M}
$$


[1] Fei Luo, Clayton F. Giese, and W. Ronald Gentry, "Direct measurement of the size of the helium dimer," J. Chem. Phys. 104, 1151 (1996).

[2] Wieland Schöllkopf and J. Peter Toennies, "The nondestructive detection of the helium dimer and trimer," J. Chem. Phys. 104, 1155 (1996).

[3] Wieland Schöllkopf and J. P. Toennies, "Nondestructive mass selection of small van der waals clusters," Science 266, 1345-1348 (1994).

[4] R. Grisenti, W. Schöllkopf, J. Toennies, G. Hegerfeldt, T. Köhler, and M. Stoll, "Determination of the bond length and binding energy of the helium dimer by diffraction from a transmission grating," Phys. Rev. Lett. 85, 2284-2287 (2000).

[5] V Efimov, "Energy levels arising from resonant two-body forces in a three-body system," Phys. Lett. B 33, 563-564 (1970).

[6] V Efimov, "Weak bound states of three resonantly interacting particles," Sov. J. Nucl. Phys. 12, 589 (1971), [Yad. Fiz. 12, 1080-1090 (1970)].

[7] Eric Braaten and H.-W. Hammer, "Universality in few-body systems with large scattering length," Physics Reports 428, 259-390 (2006).

[8] B. Esry, C. Lin, and Chris Greene, "Adiabatic hyperspherical study of the helium trimer," Phys. Rev. A 54, 394-401 (1996).

[9] P. Barletta and A. Kievsky, "Variational description of the helium trimer using correlated hyperspherical harmonic basis functions," Phys. Rev. A 64, 042514 (2001).

[10] E. Nielsen, D V Fedorov, and A S Jensen, "The structure of the atomic helium trimers: halos and efimov states," J. Phys. B 31, 4085 (1998).

[11] E. A. Kolganova, A. K. Motovilov, and W. Sandhas, "Ultracold collisions in the system of three helium atoms," Phys. Part. Nuclei 40, 206-235 (2009).

[12] A.K. Motovilov, W. Sandhas, S.A. Sofianos, and E.A. Kolganova, "Binding energies and scattering observables in the 4He3 atomic system," Eur. Phys. J. D 13, 33-41 (2001).

[13] Hiroya Suno and B. Esry, "Adiabatic hyperspherical study of triatomic helium systems," Phys. Rev. A 78, 062701 (2008).

[14] E A Kolganova, A K Motovilov, and S A Sofianos, "Three-body configuration space calculations with hard-core potentials," J. Phys. B 31, 1279 (1998). 
[15] E. Nielsen, D. V. Fedorov, A. S. Jensen, and E. Garrido, "The three-body problem with short-range interactions," Physics Reports 347, 373 - 459 (2001).

[16] Marius Lewerenz, "Structure and energetics of small helium clusters: Quantum simulations using a recent perturbational pair potential," J. Chem. Phys. 106, 4596 (1997).

[17] D. Blume and Chris H. Greene, "Monte carlo hyperspherical description of helium cluster excited states," J. Chem. Phys. 112, 8053 (2000).

[18] Javier von Stecher and Chris Greene, "Correlated gaussian hyperspherical method for fewbody systems," Phys. Rev. A 80, 022504 (2009).

[19] A. Kievsky, E. Garrido, C. Romero-Redondo, and P. Barletta, "The helium trimer with SoftCore potentials," Few-Body Syst(2011), in press.

[20] Ronald A. Aziz and Martin J. Slaman, "An examination of ab initio results for the helium potential energy curve," J. Chem. Phys. 94, 8047 (1991).

[21] Peter Lepage, "How to renormalize the schrodinger equation," (1997), arXiv:nucl-th/9706029.

[22] P. Bedaque, H.-W. Hammer, and U. van Kolck, "Renormalization of the Three-Body system with Short-Range interactions," Phys. Rev. Lett. 82, 463-467 (1999).

[23] M. Gattobigio, A. Kievsky, and M. Viviani, "Nonsymmetrized hyperspherical harmonic basis for an a-body system," Phys. Rev. C 83, 024001 (2011).

[24] H. -W. Hammer and L. Platter, "Universal properties of the four-body system with large scattering length," Eur. Phys. J. A 32, 113-120 (2007).

[25] J. von Stecher, J. P. D'Incao, and Chris H. Greene, "Signatures of universal four-body phenomena and their relation to the efimov effect," Nat Phys 5, 417-421 (2009).

[26] A. Deltuva, "Efimov physics in bosonic atom-trimer scattering," Phys. Rev. A 82, 040701(R) (2010).

[27] Javier von Stecher, "Weakly bound cluster states of efimov character," J. Phys. B: At. Mol. Opt. Phys. 43, 101002 (2010).

[28] M. Gattobigio, A. Kievsky, M. Viviani, and P. Barletta, "Harmonic hyperspherical basis for identical particles without permutational symmetry," Phys. Rev. A 79, 032513 (2009).

[29] M. Gattobigio, A. Kievsky, and M. Viviani, "Nonsymmetrized hyperspherical harmonics approach to A = 6 system," Few-Body Systems 50, 463-465 (2011).

[30] N. Ya Vilenkin, G. I. Kuznetsov, and Ya. A. Smorodinsky, "Eigenfunctions of the Laplace operator providing representations of the $\mathrm{U}(2), \mathrm{SU}(2), \mathrm{SO}(3), \mathrm{U}(3)$, and $\mathrm{SU}(3)$ groups and 
the symbolic method," Sov. J. Nucl. Phys. 2, 645-652 (1966).

[31] M. S Kil'dyushov, "Hyperspherical functions of the "Tree" type in the $n$-body problem," Sov. J. Nucl. Phys. 15, 113-117 (1972), [Yad. Fiz. 15, 197 (1972)].

[32] M. S. Kil'dyushov, "The n-body problem. Matrix elements of permutations." Sov. J. Nucl. Phys. 16, 117-119 (1973), [Yad. Fiz. 16, 217 (1972)].

[33] M. Gattobigio, A. Kievsky, M. Viviani, and P. Barletta, "Non-symmetrized basis function for identical particles," Few-Body Systems 45, 127-131 (2009). 
TABLE I. The ground state $E_{3 b}^{(0)}$ and the excited state $E_{3 b}^{(1)}$ of the helium trimer calculated with the LM2M2 potential, with its gaussian representation and with the gaussian potential plus the three-body potential. In this case the two parameters, the strength $W_{0}$ and the range $\rho_{0}$ are given.

\begin{tabular}{ccc}
\hline potential & $E_{3 b}^{(0)}(\mathrm{mK})$ & $E_{3 b}^{(1)}(\mathrm{mK})$ \\
\hline LM2M2 [11] & -126.4 & -2.265 \\
gaussian & -150.4 & -2.467 \\
\hline$\left(W_{0}(\mathrm{~K}), \rho_{0}(\right.$ a.u. $\left.)\right)$ & & \\
$(306.9,4)$ & -126.4 & -2.283 \\
$(18.314,6)$ & -126.4 & -2.287 \\
$(4.0114,8)$ & -126.4 & -2.289 \\
$(1.4742,10)$ & -126.4 & -2.292 \\
$(0.721,12)$ & -126.4 & -2.295 \\
$(0.422,14)$ & -126.4 & -2.299 \\
$(0.279,16)$ & -126.4 & -2.302 \\
\hline
\end{tabular}


TABLE II. $A=4,5,6$ binding energies of the ground, $E_{A b}^{(0)}$, and the first two excited states, $E_{A b}^{(1)}$ and $E_{A b}^{(2)}$, for increasing values of the grand-angular-quantum number $K$ using the two-body soft-core gaussian potential. We also report the symmetry of the states; the ground, $E_{A b}^{(0)}$, and the first-exited, $E_{A b}^{(1)}$, states are totally symmetric; the second-exited state belongs to a mixed representation.

\begin{tabular}{|c|c|c|c|c|c|c|c|c|c|}
\hline$K$ & $\begin{array}{c}E_{4 b}^{(0)}(m K) \\
{[4]}\end{array}$ & $\begin{array}{c}E_{4 b}^{(1)}(m K) \\
{[4]}\end{array}$ & $\begin{array}{c}E_{4 b}^{(2)}(m K) \\
{\left[\begin{array}{ll}3 & 1\end{array}\right]}\end{array}$ & $\begin{array}{c}E_{5 b}^{(0)}(m K) \\
{[5]}\end{array}$ & $\begin{array}{c}E_{5 b}^{(1)}(m K) \\
{[\mathbf{5}]}\end{array}$ & $\begin{array}{c}E_{5 b}^{(2)}(m K) \\
{\left[\begin{array}{ll}4 & 1\end{array}\right]}\end{array}$ & $\begin{array}{c}E_{6 b}^{(0)}(m K) \\
{[\mathbf{6}]}\end{array}$ & $\begin{array}{c}E_{6 b}^{(1)}(m K) \\
{[\mathbf{6}]}\end{array}$ & $\begin{array}{c}E_{6 b}^{(2)}(m K) \\
{\left[\begin{array}{ll}\mathbf{5} & \mathbf{1}\end{array}\right]}\end{array}$ \\
\hline 0 & 725.98 & 31.688 & & 1913.0 & 642.84 & & 3773.1 & 2010.7 & \\
\hline 2 & 725.98 & 31.688 & & 1913.0 & 642.84 & 314.15 & 3773.1 & 2010.9 & 1626.5 \\
\hline 4 & 746.45 & 77.971 & & 1941.2 & 746.01 & 400.95 & 3807.6 & 2140.1 & 1719.3 \\
\hline 6 & 750.15 & 107.63 & & 1944.1 & 778.79 & 516.60 & 3809.9 & 2166.2 & 1840.5 \\
\hline 8 & 751.06 & 124.48 & 2.5177 & 1945.0 & 802.47 & 571.03 & 3810.8 & 2188.6 & 1882.5 \\
\hline 10 & 751.28 & 135.94 & 29.401 & 1945.2 & 813.88 & 608.58 & 3810.9 & 2196.4 & 1909.0 \\
\hline 12 & 751.35 & 144.17 & 50.336 & 1945.2 & 820.87 & 634.25 & 3810.9 & 2200.8 & 1923.4 \\
\hline 14 & 751.37 & 149.30 & 66.672 & 1945.2 & 824.84 & 653.19 & 3810.9 & 2202.7 & 1931.9 \\
\hline 16 & 751.37 & 152.98 & 79.082 & 1945.2 & 827.23 & 657.59 & 3810.9 & 2203.6 & 1936.5 \\
\hline 18 & 751.38 & 155.54 & 89.069 & 1945.2 & 828.67 & 678.86 & 3810.9 & 2204.0 & 1938.7 \\
\hline 20 & 751.38 & 157.43 & 97.021 & 1945.2 & 829.58 & 687.87 & 3810.9 & 2204.1 & 1939.7 \\
\hline 22 & 751.38 & 158.76 & 103.54 & 1945.2 & 830.15 & 695.23 & 3810.9 & 2204.2 & 1940.0 \\
\hline 24 & 751.38 & 159.77 & 108.90 & 1945.2 & 830.50 & 701.29 & & & \\
\hline 26 & 751.38 & 160.53 & 113.38 & & & & & & \\
\hline 28 & 751.38 & 161.10 & 117.16 & & & & & & \\
\hline 30 & 751.38 & 161.54 & 120.37 & & & & & & \\
\hline 32 & 751.38 & 161.89 & 123.13 & & & & & & \\
\hline 34 & 751.38 & 162.15 & 125.52 & & & & & & \\
\hline 36 & 751.38 & 162.37 & 127.60 & & & & & & \\
\hline 38 & 751.38 & 162.53 & 129.42 & & & & & & \\
\hline 40 & 751.38 & 162.67 & 131.02 & & & & & & \\
\hline
\end{tabular}


TABLE III. $A=4,5,6$ binding energies of the ground, $E_{A b}^{(0)}$, and first-excited, $E_{A b}^{(1)}$, states for increasing values of the grand-angular-quantum number $K$. The three-body force parameters are $\rho_{0}=14$ a.u. and $W_{0}=0.422 \mathrm{~K}$. We also report the symmetry of the states; both the ground, $E_{A b}^{(0)}$, and the first-exited, $E_{A b}^{(1)}$, states are totally symmetric.

\begin{tabular}{|c|c|c|c|c|c|c|}
\hline$K$ & $\begin{array}{c}E_{4 b}^{(0)}(\mathrm{mK}) \\
{[4]}\end{array}$ & $\begin{array}{c}E_{4 b}^{(1)}(\mathrm{mK}) \\
{[4]}\end{array}$ & $\begin{array}{c}E_{5 b}^{(0)}(\mathrm{mK}) \\
{[5]}\end{array}$ & $\begin{array}{c}E_{5 b}^{(1)}(\mathrm{mK}) \\
{[5]}\end{array}$ & $\begin{array}{c}E_{6 b}^{(0)}(\mathrm{mK}) \\
{[6]}\end{array}$ & $\begin{array}{c}E_{6 b}^{(1)}(\mathrm{mK}) \\
{[\mathbf{6}]}\end{array}$ \\
\hline 0 & 538.93 & 4.557 & 1288.1 & 365.1 & 2293.8 & 1109.9 \\
\hline 2 & 538.93 & 4.557 & 1288.1 & 365.1 & 2293.8 & 1109.9 \\
\hline 4 & 561.69 & 40.29 & 1319.6 & 460.4 & 2331.8 & 1237.3 \\
\hline 6 & 566.68 & 67.47 & 1324.4 & 497.6 & 2336.6 & 1273.0 \\
\hline 8 & 568.21 & 84.22 & 1326.1 & 527.0 & 2338.4 & 1307.7 \\
\hline 10 & 568.58 & 96.04 & 1326.5 & 542.7 & 2338.7 & 1323.1 \\
\hline 12 & 568.73 & 105.30 & 1326.6 & 554.0 & 2338.8 & 1334.4 \\
\hline 14 & 568.77 & 111.17 & 1326.6 & 561.0 & 2338.9 & 1340.9 \\
\hline 16 & 568.78 & 115.58 & 1326.6 & 565.9 & 2338.9 & 1345.3 \\
\hline 18 & 568.79 & 118.78 & 1326.6 & 569.3 & 2338.9 & 1348.2 \\
\hline 20 & 568.79 & 121.20 & 1326.6 & 571.8 & 2338.9 & 1350.2 \\
\hline 22 & 568.79 & 122.98 & 1326.6 & 573.6 & 2338.9 & 1351.6 \\
\hline 24 & 568.79 & 124.38 & 1326.6 & 574.9 & & \\
\hline 26 & 568.79 & 125.47 & & & & \\
\hline 28 & 568.79 & 126.33 & & & & \\
\hline 30 & 568.79 & 127.02 & & & & \\
\hline 32 & 568.79 & 127.57 & & & & \\
\hline 34 & 568.79 & 128.02 & & & & \\
\hline 36 & 568.79 & 128.40 & & & & \\
\hline 38 & 568.79 & 128.70 & & & & \\
\hline 40 & 568.79 & 128.96 & & & & \\
\hline Ref.[16] & 558.4 & & 1302.2 & & 2319.4 & \\
\hline Ref.[17] & 559.7 & 132.6 & 1309.3 & 597.1 & 2329.4 & 1346.7 \\
\hline
\end{tabular}


TABLE IV. The ratios $E_{A b}^{(0)} / E_{3 b}^{(0)}$ and $E_{A b}^{(1)} / E_{(A-1) b}^{(0)}$ as a function of the three-body cutoff $\rho_{0}$.

\begin{tabular}{ccccccc}
\hline$\rho_{0}$ (a.u.) & $E_{4 b}^{(0)} / E_{3 b}^{(0)}$ & $E_{4 b}^{(1)} / E_{3 b}^{(0)}$ & $E_{5 b}^{(0)} / E_{3 b}^{(0)}$ & $E_{5 b}^{(1)} / E_{4 b}^{(0)}$ & $E_{6 b}^{(0)} / E_{3 b}^{(0)}$ & $E_{6 b}^{(1)} / E_{5 b}^{(0)}$ \\
\hline 12 & 4.47 & 1.01 & 10.33 & 1.001 & 18.12 & 1.005 \\
14 & 4.50 & 1.02 & 10.50 & 1.011 & 18.50 & 1.018 \\
16 & 4.54 & 1.03 & 10.70 & 1.021 & 19.06 & 1.029 \\
\hline
\end{tabular}




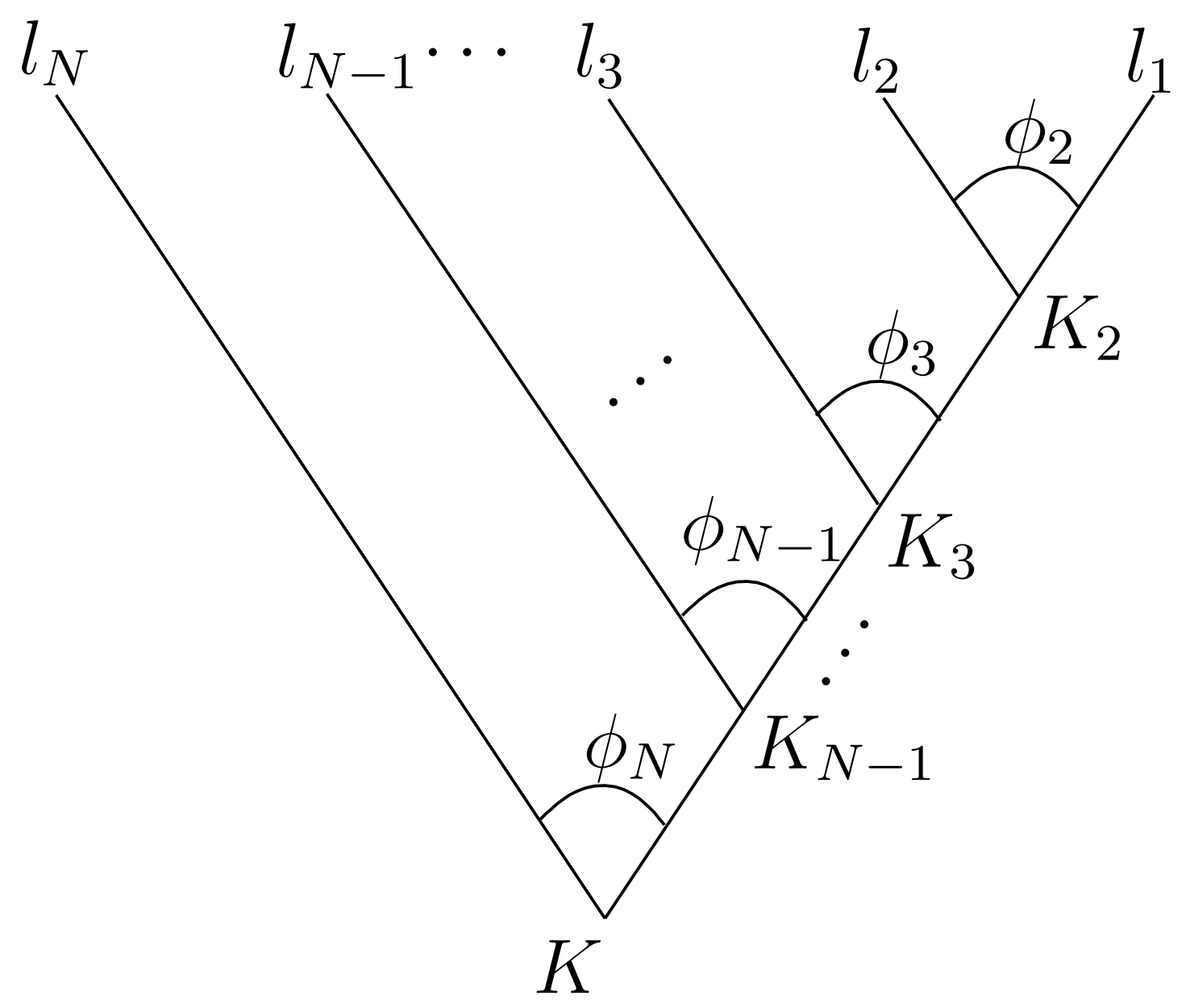

FIG. 1. Hyperspherical tree corresponding to Eq.(9) 

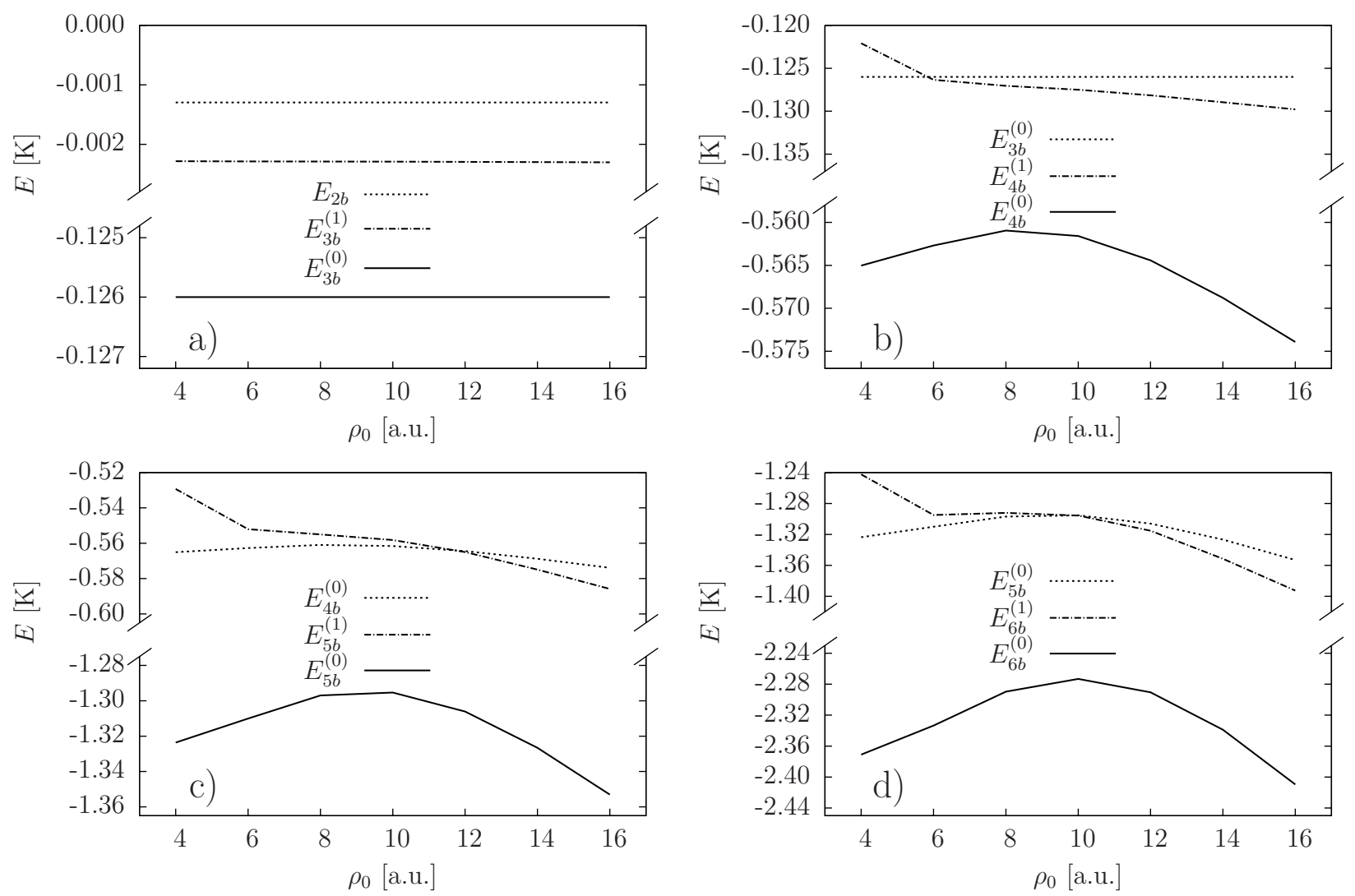

FIG. 2. Ground- and excited-state energies of the $A=2,3,4,5,6$ systems as a function of $\rho_{0}$. In panel a) we report the ground- and excited-state energy of $A=3$ system together with the ground-state energy of $A=2$; for all of the values of $\rho_{0}$ we have considered, the excited $A=3$ state is bounded. In panel b) we report the ground- and excited-state energy of $A=4$ system together with the ground-state energy of $A=3$; the excited $A=4$ state is bounded for $\rho_{0}>7$. In panel c) we report the ground- and excited-state energy of $A=5$ system together with the ground-state energy of $A=4$; the excited $A=5$ state is bounded for $\rho_{0}>12$. In panel d) we report the ground- and excited-state energy of $A=6$ system together with the ground-state energy of $A=5$; the excited $A=6$ state is bounded for $\rho_{0}>10$. 